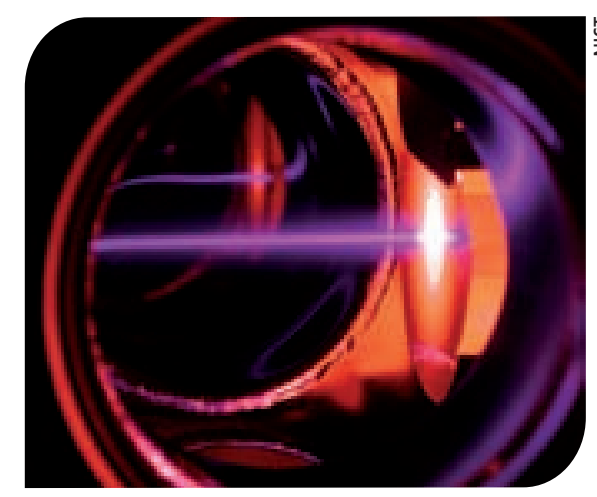

Figure 4. Faisceau laser violet ralentissant des atomes d'Erbium à la sortie d'un four à $1300^{\circ} \mathrm{C}$ au « National Institute of Standards and Technology ".

et ions tels que le sodium $\mathrm{Na}$ (à $599 \mathrm{~nm}$ ), et I'Ytterbium Yb (à $399 \mathrm{~nm}$ ). Les techniques de génération d'harmoniques permettent de combler ces " trous " par doublage, quadruplage, somme ou différence de fréquence.

Afin d'augmenter l'efficacité de conversion, le cristal non linéaire est intégré dans une cavité résonnante de type «bow-tie» stabilisée activement sur la longueur d'onde du fondamental. Comparées à d'autres technologies, ces solutions à semi-conducteurs n'utilisent ni laser de pompe, ni refroidissement à eau. Ces systèmes sont compacts, fiables et très simples d'utilisation.

\section{Applications}

Les DLCE trouvent leur application et sont utilisées de manière routinière dans un nombre impressionnant de domaines, sans être exhaustif nous citons les principales :

- refroidissement et piégeage d'atomes neutres: $\mathrm{He}^{*}, \mathrm{Li}, \mathrm{Ne}^{*}, \mathrm{Na}, \mathrm{Mg}, \mathrm{Al}, \mathrm{Ar}^{*}$, $\mathrm{K}, \mathrm{Ca}, \mathrm{Cr}, \mathrm{Fe}, \mathrm{Ga}, \mathrm{Kr}, \mathrm{Rb}, \mathrm{Sr}, \mathrm{Ag}, \mathrm{Cd}$, In, $\mathrm{Xe}, \mathrm{Cs}, \mathrm{Ba}, \mathrm{Dy}, \mathrm{Er}, \mathrm{Tm}, \mathrm{Yb}, \mathrm{Hg}, \mathrm{Fr}, \mathrm{Ra}$ - refroidissement et piégeage d'ions - obtention de la condensation de BoseEinstein et de gaz de Fermi dégénérés - horloge à fontaine atomique.

\title{
Les lasers à fibre
}

\section{$\gg$ David PUREUR, \\ Alexandre BIASI \\ Quantel SA, Lannion \\ alexandre.biasi@quantel.fr www.quantel-laser.com}

$\mathrm{P}$ our certaines applications liées aux atomes froids, le laser à fibre est utilisé et il présente des avantages évidents en termes de coûts, compacité, flexibilité et simplicité d'utilisation. Les sources les plus communément utilisées sont des lasers à fibre émettant une radiation dans I'infrarouge à 1064 nm, notamment pour la réalisation de pièges dipolaires optiques (qui permettent de concentrer et sélectionner les atomes les plus froids par évaporation afin de ne conserver que les atomes ayant des températures de l'ordre de quelques fractions de microkelvins). Ces lasers qui émettent hors de la bande d'absorption des atomes refroidis, ont une puissance continue qui peut aller jusqu'à plusieurs dizaines de watts pour une largeur de raie spectrale inférieure au $\mathrm{MHz}$. Les spécifications en bruit d'intensité du laser, mais aussi en stabilité du pointé de faisceau sont importantes afin de minimiser les fluctuations et assurer par exemple une bonne stabilisation du condensat de Bose-Einstein étudié. D'autres longueurs d'onde peuvent également être utilisées autour de 1.56 microns avec des lasers à fibre émettant en régime continu une puissance de quelques dizaines de watts. De tels lasers IR sont une alternative intéressante aux lasers $\mathrm{CO}_{2}$ pour certaines étapes du refroidissement.

Une autre application intéressante autour de laquelle les sources fibrées se développent est le ralentissement des atomes au sein des pièges magnéto-optiques (MOT-2D et 3D). Ces pièges assurent le refroidissement des atomes de quelques millikelvins à quelques dizaines de microkelvins.

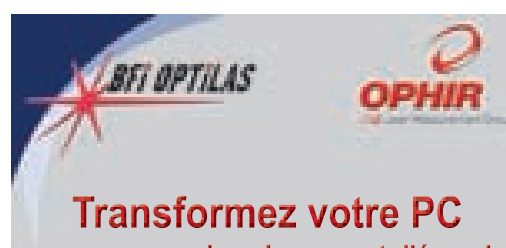

en mesureur de puissance et d'énergie

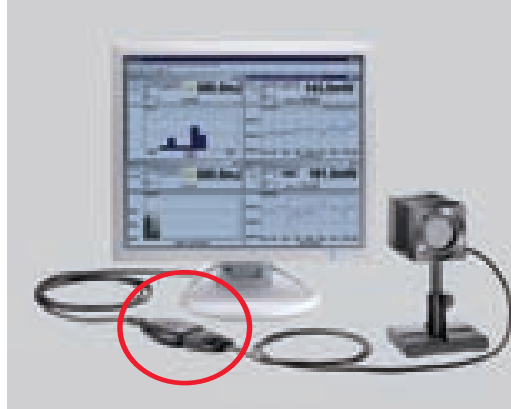

Nouvelle interface USB compacte permettant la mesure de la puissance et de l'énergie sans afficheur, directement sur votre PC.

- connexion facile

- compacte

- compatible avec toutes les têtes de mesure de puissance et d'énergie OPHIR

- taux de répétition jusqu'à $10 \mathrm{kHz}$ avec des capteurs pyro-électriques
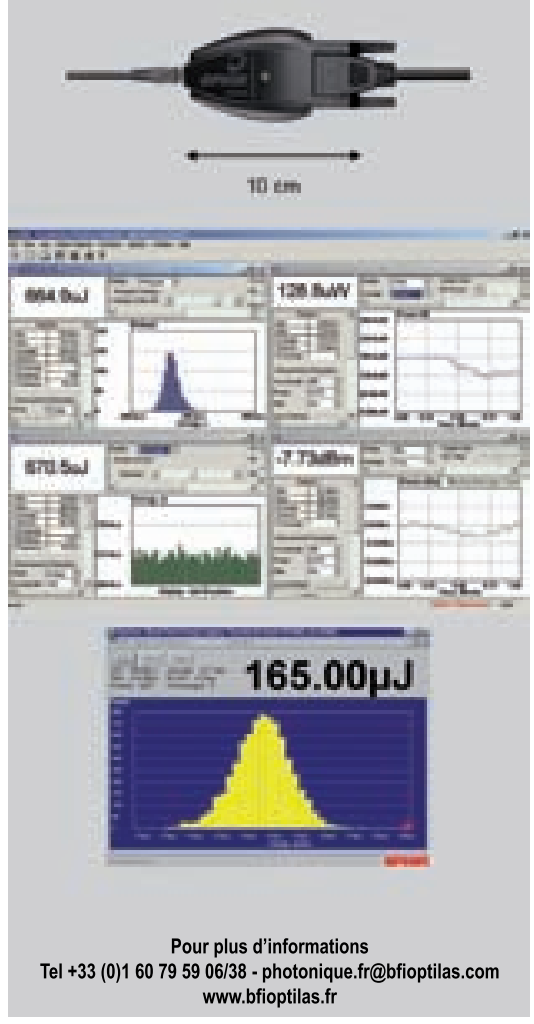
Jusqu'à aujourd'hui, les solutions privilégiées sont à base de sources à semiconducteurs et de cavités externes.

Plusieurs publications depuis 2003, dont une de l'équipe française Optique Atomique de I'Institut d'Optique [1], ont démontré la possibilité de refroidir des atomes avec un laser à fibre émettant un rayonnement visible à $780 \mathrm{~nm}$ (transition D2 de l'ion rubidium). La solution repose sur l'amplification par différents étages d'amplification fibrés d'un laser à semi-conducteur de type DFB émettant à 1,56 micron (de $10 \mathrm{kHz}$ à $1 \mathrm{MHz}$ de largeur de raie spectrale, selon les fournisseurs). La longueur d'onde infrarouge est ensuite doublée en simple passage dans un cristal dit périodiquement polarisé (tel le PPLN). Des puissances de plusieurs watts ont été démontrées à $780 \mathrm{~nm}$ dans la littérature avec pour certaines architectures une largeur de raie spectrale inférieure à $100 \mathrm{kHz}$.

L'avantage d'une telle solution est d'a bord I'utilisation de nombreux composants issus du secteur des télécoms : le laser maître (de faible puissance) est en effet une diode laser DFB émettant à 1,56 micron suffisamment fine spectralement pour pouvoir être utilisée sans cavité externe (d'où l'excellente stabilité obtenue avec aucun alignement susceptible d'être perturbé). Le laser ainsi conçu profite ensuite des nombreux avantages de l'amplification à fibre qui associe robustesse, maîtrise de la largeur de raie et conservation de la polarisation linéaire du faisceau. Les cristaux doubleurs utilisés ont fait d'énormes progrès et il est désormais possible d'avoir des efficacités de conversion IR-visible de l'ordre de $20 \%$ (en régime monofréquence). Par ailleurs, les techniques de codopage développées par les fabricants de cristaux ont permis de réduire fortement les phénomènes photochromiques et photoréfractifs initialement présents au sein de ces cristaux. Des durées de vie de plusieurs milliers d'heures ont ainsi été démontrées avec des cristaux supportant une puissance visible supérieure à $5 \mathrm{~W}$ (à $532 \mathrm{~nm}$ ). L'architecture à fibre est également suffisamment flexible pour intégrer des composants de type acousto-optique et électro-optique au sein du laser pour accéder ainsi, avec le minimum de pertes, à toutes les fonctionnalités spectrales et spatiales nécessaires aux dispositifs de refroidissement d'atomes : obturation rapide des faisceaux, décalage ou création de bandes latérales pour les systèmes de repompage optique ou d'imagerie, accordabilité en longueur d'onde...

L'atome de rubidium étant le plus étudié, la longueur d'onde de $780 \mathrm{~nm}$ est très utilisée dans les laboratoires. L'atome de potassium est quant à lui refroidi avec un faisceau à la longueur d'onde de $767 \mathrm{~nm}$ et plusieurs équipes travaillent sur la réalisation de lasers à fibre à cette longueur d'onde. Cet atome présente en effet un intérêt pour la gravimétrie et les applications spatiales. Obtenir de la puissance à $767 \mathrm{~nm}$ nécessite d'abord l'utilisation d'une source fibrée à $1534 \mathrm{~nm}$ (quelques watts). Bien que cela corresponde à une longueur d'onde employée en télécom, il existe quelques difficultés techniques liées à la présence de phosphore dans les fibres double gaine erbium/ytterbium (nécessaire à l'obtention de puissance), ce qui réduit la bande spectrale d'amplification accessible. Des solutions basées sur l'utilisation de fibres dopées à l'erbium seuls ou sur de l'amplification Raman sont à l'étude.

La longueur d'onde de $589 \mathrm{~nm}$ est aussi employée pour le refroidissement des atomes de sodium. Cette espèce atomique présente en effet des interactions entre états de spin très intéressantes à la fois par leur nature (antiferromagnétique) et par leur intensité, significativement plus importante que celle observée pour le rubidium plus communément employé. Plusieurs équipes travaillent sur cette espèce pour la réalisation de

\section{Synthèse}

\begin{tabular}{|l|l|l|}
\hline Longueurs d'onde disponibles & de $205 \mathrm{~nm}$ à $1670 \mathrm{~nm}$ & 589,780 et $766.5 \mathrm{~nm}$ \\
\hline Accordabilité avec saut de mode & de 1 à $60 \mathrm{~nm}$ & de $0.3 \mathrm{~nm}$ à $0.8 \mathrm{~nm}$ \\
\hline Accordabilité sans saut de mode & de 20 à $50 \mathrm{GHz}$ & de 20 à $200 \mathrm{GHz}$ \\
\hline Finesse spectrale & de $1 \mathrm{MHz}$ à $1 \mathrm{~Hz}$ & de $1,5 \mathrm{MHz}$ à $50 \mathrm{kHz}$ \\
\hline Qualité de faisceau (M²) & 1,2 & 1,1 \\
\hline Puissance & jusqu'à $2 \mathrm{~W}$ & jusqu'à $20 \mathrm{~W}$ \\
\hline
\end{tabular}

condensats de Bose-Einstein et l'observation d'états quantiques de spin fortement corrélés [2]. Pour une application en astronomie (création d'étoiles artificielles), une équipe de l'ESO [3] a démontré une source fibrée basée à la fois sur de l'amplification ytterbium (lasers de pompe continus émettant autour de $1070 \mathrm{~nm}$ ) et de l'amplification Raman pour décaler le spectre optique et obtenir une source IR de puissance à $1178 \mathrm{~nm}$. Plus de $60 \mathrm{~W}$ ont ainsi été reportés dans la littérature. Le caractère monofréquence (la largeur de raie spectrale est inférieure à $7 \mathrm{MHz}$ ) de ce laser est en outre compatible avec un doublage en cavité résonante massive (avec un cristal de LBO) et une puissance de $14,5 \mathrm{~W}$ à $589 \mathrm{~nm}$ a été démontrée. Le refroidissement d'atome ne nécessite pas autant de puissance à $589 \mathrm{~nm} ; 500 \mathrm{~mW}$ à $1 \mathrm{~W}$ sont généralement suffisants.

En résumé, les sources à fibre de puissance émettant dans l'infrarouge proche sont très présentes au sein des laboratoires réalisant le refroidissement d'atomes. L'association de l'amplification fibrée de puissance avec une nouvelle génération de cristaux doubleurs permet aujourd'hui à cette technologie de se positionner sur le secteur des sources visibles cohérentes $(780 \mathrm{~nm}, 767 \mathrm{~nm}, 589 \mathrm{~nm}$ mais aussi $532 \mathrm{~nm}, 577 \mathrm{~nm}, 633 \mathrm{~nm}$...) en régime continu.

\section{Références \\ [1] Équipe d'optique atomique, Laboratoire C. Fabry, Institut d'Optique Graduate School \\ [2] Équipe micro-condensats, Laboratoire K. Brossel \\ [3] L. Taylor et al., « High power narrowband 589 nm frequency doubled fibre laser source $», 0$ ptic Express vol. 17 (17), 2009.}

